\title{
INFLUÊNCIA DO ASSENTO DA CADEIRA ADAPTADA NA EXECUÇÃO DE UMA TAREFA DE MANUSEIO
}

INFLUENCE OF THE SEAT SURFACE OF AN ADAPTED CHAIR ON THE PERFORM ANCE OF A MANIPULATION TASK

\author{
Lígia Maria Presumido BRACCIALLI ${ }^{1}$ \\ Franciane Teixeira de OLIVEIRA ${ }^{2}$ \\ A na CarlaBRACCIALLI ${ }^{3}$ \\ Andréia Naomi SANKAKO ${ }^{4}$
}

\begin{abstract}
RESUM O: este estudo teve como objetivo verificar a influência da flexibilidade da superfície de assento da cadeira na velocidade e no tempo despendido por alunos com paralisia cerebral espástica durante a execução de uma tarefa de manuseio de um objeto na posição sentada. Participaram do estudo 11 alunos, de ambos os gêneros, com diagnóstico de paralisia cerebral espástica, que tinham algum controle de tronco e membros superiores. A quantificação da análise cinemática foi realizada em duas situações experimentais: 1) execução de uma tarefa acadêmica de encaixe, com o indivíduo posicionado em um mobiliário adaptado com assento de Iona; 2) execução de uma tarefa acadêmica de encaixe, com o participante posicionado em um mobiliário adaptado com assento de madeira. Os dados obtidos foram submetidos à análise estatística descritiva e nãoparamétrica por meio do teste de Wilcoxon. Os resultados indicaram que: 1) a velocidade média de execução das tarefas não foi influenciada pelo tipo de assento utilizado 2) o tempo de execução da tarefa foi influenciada pelo tipo de assento utilizado. A utilização do assento de lona aumentou o tempo de realização da tarefa. Conclui-se que o assento de um mobiliário escolar para um aluno com paral isia cerebral espástica não deve ser confeccionado com um material muito flexível. Este tipo de assento fornecerá uma base instável que dificultará o desempenho do aluno durante atividades realizadas com os membros superiores.
\end{abstract}

PALAVRAS-CHAVE: mobiliário escolar; paralisia cerebral; tecnologia assistiva; educação especial.

\begin{abstract}
A BSTRACT: the objective of this study was to verify the influence of the flexibility of the seat surface of a chair on the speed and time expended by students with spastic cerebral palsy during the performance of a task involving the manipulation an object while in a seated position. Eleven students of both genders participated in the study; all were diagnosed with spastic cerebral palsy, and all had some control of trunk and upper limbs. The quantification of the kinematics analysis was done through two experimental situations: 1) performance of an academic task involving fitting blocks into matching holes, with the individual seated in an adapted seat with canvas seat surface; 2) performance of an academic task involving fitting blocks into matching holes, with the participant seated in an adapted seat with wooden seat surface. The data collected was submitted to descriptive analysis and non-parametric statistics using the Wilcoxon test. The results indicated that: 1) the average speed of performance of the tasks was not influenced by the type of seat surface 2) performance time for the task was influenced by the type of seat surface. The use of the canvas seat surface increased the accomplishment time for the task. This study concluded that the seat surface of school furniture for students with spastic cerebral palsy should not to be made from very flexible materials. This type of seat surface does not offer the necessary stability, and will be detrimental to thestudent's performance during activities involving the upper limbs.
\end{abstract}

KEYWORDS: school furniture; cerebral palsy; assistive technology; special education.

${ }^{1}$ Fisioterapeuta, Doutora em Educação Física pela Unicamp, Docente do curso de fisioterapia da Unesp-MaríliaSP, Departamento de Educação Especial. - bracci@marilia.unesp.br (A poio financeiro: CNPq/ CAPES/ PROESP/ MEC/ SEESP / Fundo de Pesquisa da FFC/ Marília)

${ }^{2}$ Fisioterapeuta, Mestranda em Educação na Unesp-Marília-SP, Bolsista CAPES/ PROESP/ SEESP/ MEC. fisiofran2003@yahoo.com.br

${ }^{3}$ Aluna do $2^{\circ}$ ano de fisioterapia da Unesp-Marília-SP. - karlinhaaa@hotmail.com

${ }^{4}$ Fisioterapeuta, mestranda em Educação na Unesp-Marília-SP. Bolsista CNPq. - asankako@yahoo.com.br 


\section{INTRODUÇÃO}

A pesar deindiscutível a importância da aquisição edo uso detecnologia assistiva para mel horar a interação social, o desempenho ea qualidade de vida da pessoa com deficiência, vários estud os têm mostrado que mais de $30 \%$ detodos os dispositivos adquiridos foram abandonados pelo usuário entre o primeiro ano eo quinto ano de uso, e al guns não chegaram nem mesmo a ser utilizado (PHILLIPS; ZHAO, 1993; GOODMAN; TIENE; LUFT, 2002; SCHERER, 2002; VERZA et al., 2006). Estes autores relataram vários motivos que levaram o usuário a abandonar o recurso prescrito eadquirido: 1) fal ta de participação do usuário durante a seleção do dispositivo; 2) desempenho ineficaz do dispositivo; 3) mudanças nas necessidades do usuário; 4) falta do treinamento do usuário; 5) dispositivo inadequado às necessidades do usuário; 6) dispositivos de uso complicado; 7) aceitação social do dispositivo; 8) fal ta de motivação; para o uso do dispositivo; 9) falta de treinamento e conhecimento do dispositivo; 10) dispositivo com aparência, peso e tamanho não-estéticos.

A possibilidade de abandono de um dispositivo diminui quando se conhece em detal he sua funcionalidade e a influência no desempenho funcional dos usuários.

Assim, vários estudos têm sido realizados para verificar como a cadeira adaptada prescrita para a criança com paralisia cerebral pode interferir no desempenho funcional deste usuário.

A cadeira confeccionada para a criança com paralisia cerebral deve compensar a falta de estabilidade postural, aperfeiçoar as habilidades funcionaise mel horar o potencial do usuário (GREEN ; NELHAM, 1991; NWA OBI, 1987; MYHR; VON WENDT, 1991; MCCLENAGHAN; THOMBS; MILNER, 1992).

A preservação da curvatura lombar e a estabilização pélvica são fundamentais para a manutenção de um alinhamento postural adequado. Desta forma, a cadeira adaptada para a criança com paralisia cerebral espástica deve dispor de recursos que favoreça esse alinhamento (BRACCIALLI, 2000).

A pelve é o segmento chave para um sentar efetivo para crianças com paralisia cerebral quando se ressalta a função de membros superiores (REID; SOCHANIWSKYJ; MILNER,1992).

A pel ve desempenha importantepapel na manutenção da estática eda dinâmica do corpo. As cristas do osso ilíaco funcionam como braço de alavanca para as cadeias musculares do tronco e, também, para as cadeias musculares dos membros inferiores. Dessa forma, sendo a pelve o elo entre a parte superior e a inferior do corpo, qualquer modificação em sua biomecânica resultará em compensações em outras estruturas do corpo.

Desta forma, o assento da cadeira é um fator importante a ser considerado durante a prescrição de uma cadeira. O assento flexível pode causar 
instabilidade postural einabilidaded o uso de membros superiores (KOCHHAN N; CANALI; SERAFIM, 2004).

Um assento adequado contribui ativamente para o desenvolvimento do controle postural da criança com paralisia cerebral. O assento é considerado parte integrante no auxílio do controle postural sentado de uma criança com paralisia cerebral (GREEN; NELHAM, 1991).

Não existe evidência de benefícios na função dos membros superiores quando os indivíduos com paral isia cerebral sentavam-seem cadeiras com assentos com inclinações superiores a 90 graus de quadril (SEEGER; CAUDREY; O'MARA, 1984).

A atividade tônica dos músculos espásticos é menor quando o corpo é colocado em posição reta secomparado com a posição reclinada, ou seja, a ind inação do encosto da cadeira dever ser mantida em $90^{\circ}$ e o assento em 0 . . Relataram, também, queesse posicionamento éo quepermitemel hor desempenho eem menor tempo de atividades funcionais real izadas com os membros superiores (NWA OBI et al., 1983; NWA OBI, 1987).

A criança tem uma melhora significativa no controle de cabeça, de tronco e pés, e nas funções dos braços e mãos quando sentadas com seu tronco superior anterior ao fulcro da tuberosidade isquiática. A inclinação anterior do assento possibilitou a restauração da curvatura lombar e facilitou a anteversão pélvica (MYHR; VON WENDT, 1990; 1991; 1993).

A inclinação anterior do tronco podefacilitar a restauração da curvatura lombar, crucial para que ocorra uma apropriada distribuição de peso na tuberosidade isquiática e mel horar a qualidade dos movimentos funcionais dos membros superiores (REID, 1996).

A textura do assento pode influenciar no alinhamento postural e na atividade funcional de membros superiores em crianças com alterações motoras (WASHINGTON et al; 2002). Os autores relataram que o assento de espuma proporcionou uma superfície não escorregadia, em contraste com o assento de vinil. Os autores verificaram que a textura do assento interferiu no alinhamento postural e na função de membros superiores.

Um assento de cadeira com uma almofada adequada pode contribuir para uma postura equilibrada e funcional e, conseqüentemente, para um melhor desempenho de membros superiores (EITZEN , 2004).

A pesar de, nos últimos anos, vários autores terem estudado a importância da adaptação do mobiliário para o desempenho funcional do usuário, poucos estudos têm abordado como a flexibilidade do assento da cadeira pode interferir na dinâmica postural do al uno com paralisia cerebral.

O estudo teve como objetivo verificar a influência da flexibilidade da superfície de assento da cadeira na velocidade e no tempo despendido por alunos 
com paralisia cerebral espástica durantea execução de uma tarefa demanuseio de um objeto na posição sentada.

\section{MÉTOdo}

O estudo foi submeti do a apreciação do Comitê de Ética e Pesquisa da Faculdade de Filosofia e Ciências - Professor Júlio de Mesquita Filho - UNESP de Marília, e obteve parecer favorável com o número 2801/ 2005.

A pesquisa foi desenvolvida no Laboratório deA nálise do M ovimento (LABAM) da Faculdade de Filosofia e Ciências (FFC), Unesp, Campus de Marília.

\subsection{Participantes}

Foram participantes do estudo onzeindivíduos do gênero masculino e feminino, na faixa etária entre 7 e 28 anos, com diagnóstico de paralisia cerebral espástica. Todos os partici pantes foram esclarecidos sobre os objetivos propostos e a importância da sua participação. Participaram do estudo, apenas aqueles indivíduos ou seus responsáveis que assinaram o Termo de Consentimento Livre elnformado.

A dotaram-se os seguintes critérios para inclusão dos participantes no estudo: 1) apresentar quadro de espasticidade; 2) possuir habilidade para segurar e deslocar um objeto pré-determinado; 3) compreender ordens simples. Foram excluídos os indivíduos queapresentaram déficits acentuados deacuidadevisual.

\subsection{ConfECÇÃo do MOBILIÁRIO ADAPTADO}

Para a real ização do estudo foi necessária a confecção de um mobiliário adaptado. A ntes da confecção do mobiliário foram sel ecionad os os 11 participantes com paralisia cerebral espástica que participariam do estudo. Esses participantes foram avaliados física eantropométricamente para o estabel ecimento de parâmetros para a construção do mobiliário utilizado na coleta de dados.

A pós essa etapa, foi construída uma cadeira demadeira que permitiaa regulagem de altura para o apoio de pés e para a profundidade de assento. A adequação da cadeira foi realizada de acordo com as medidas antropométricas de cada usuário, por meio dos ajustes existentes (Figura 1). Em relação ao assento, a cadeira foi confeccionada com um assento fixo de lona. Sobre este assento era possível encaixar um assento móvel de madeira. 


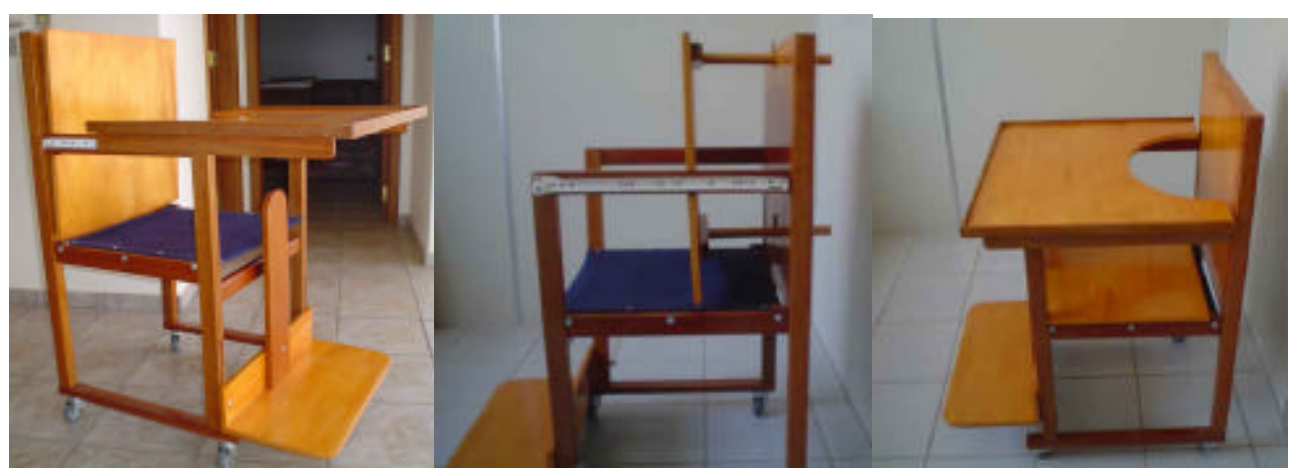

Figura 1 - Cadeira adaptada com ajustes antropométricos e assento removível.

O assento fixo de lona, como pode ser observado na Figura 1 (duas primeiras fotos) era semelhante ao utilizado em cadeiras de rodas convencional. O assento demadeira era removível eencaixado em cima do assento delona (última foto da Figura 1), durante a situação experimental de coleta de dados.

\subsection{SituAÇão EXPERIMENTAL}

O voluntário era posicionado na cadeira após ser realizado o ajuste da cadeira as suas condições antropométricas.

O inicio de cada situação experimental foi precedido por um tempo de 3 minutos. Essetempo de repouso antes do início da atividade, após a mudança da superfície de apoio, foi necessário para que ocorresse a acomodação postural na posição sentada (NWA OBI, 1987; MYHR et al., 1995). O estudo foi realizado em 2 situações experimentais: 1) realização da atividade na cadeira com o assento rígido de madei ra; 2) realização da atividade na cadeira com o assento flexível de lona.

\section{Assento RígIDO de madeira}

A pós o participante ser posicionado adequadamente na cadeira adaptada ele era orientado a realizar a tarefa com o membro superior queeletivesse maior habilidade.

A tarefa consistiu em deslocar e realizar o encaixe de um objeto de formato cilíndrico, colocado sobre um ponto pré-determinado na mesa adaptada. Os participantes foram orientados a segurar o objeto de forma a comprimi-lo, e deslocá-lo da marca inicial para outra previamente determinada.

Na mesa foram demarcados dois pontos distintos: 1) inicial e2) final. O ponto determinado como inicial estava a $15 \mathrm{~cm}$ do tronco do participante, paralelo ao eixo esternal anterior, o ponto final estava paralelo ao inicial a uma distância variável para cada participante. A distância entre o ponto inicial e final variava 
dependendo do alcance máximo transverso de cada participante. O alcancemáximo transverso é a mensuração antropométrica que determina o eixo hipotético do membro superior centrado na articulação do ombro ea partir desse ponto desenhase um raio que é igual ao comprimento do membro superior. A determinação do alcance máximo é utilizada para definir o espaço necessário para o trabalho ou atividade (NOWAK,1996; JAROSZ, 1996).

O participante foi orientado a deslocar o objeto: 1) do ponto inicial até o ponto central - foi denominando movimento de ida (Figura 2); 2) do ponto central até o ponto inicial - foi denominado movimento de retorno (Figura 3).

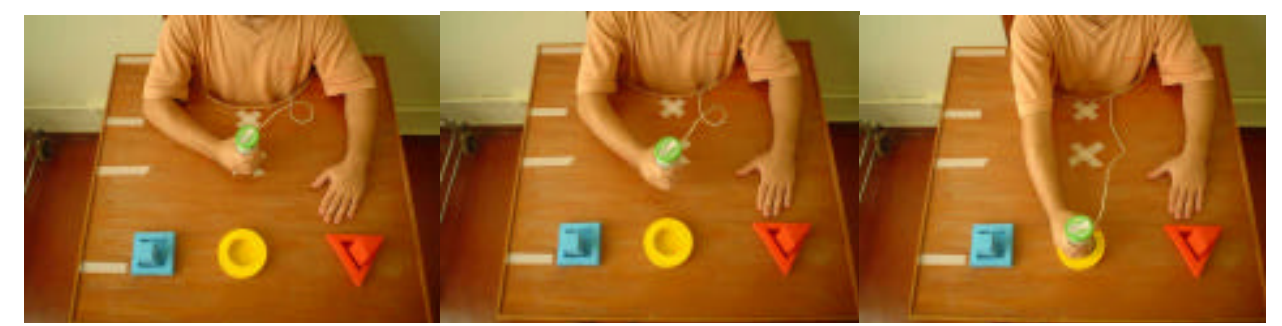

Figura 2 - Movimento de ida, deslocamento do objeto do ponto inicial ao ponto central.

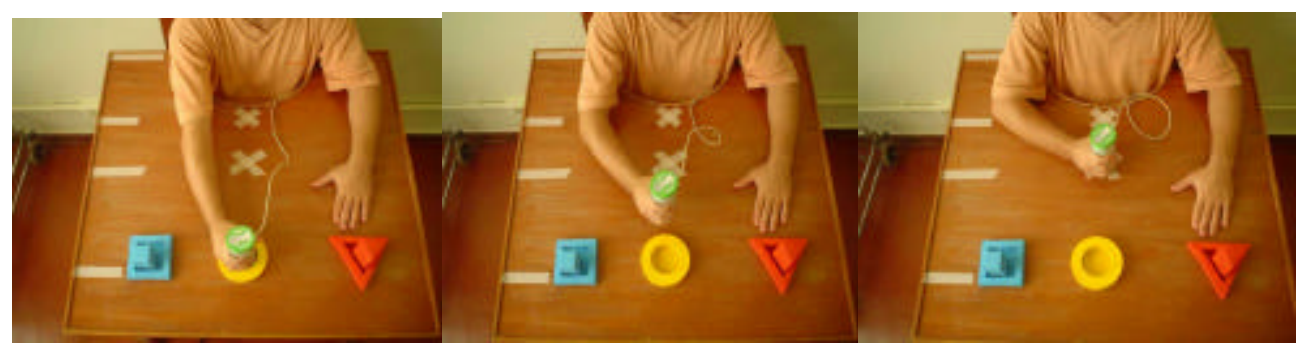

Figura 3 - Movimento de retorno, deslocamento do objeto do ponto central até o inicial.

\section{Assento flexível de Lona}

O participante era posicionado na cadeira com o assento flexível de Iona e o mesmo procedimento era realizado.

O estudo foi randômico, por sorteio, o qual era realizado antes da coleta de dados e anotado na ficha do participante, para que fosse diminuída a porcentagem de erros, e para que os possíveis erros que pudessem ocorrer não se devessem à aprendizagem da tarefa em determinado tipo deassento (NWAOBI et al., 1983). 


\subsection{Registro de dados}

Para o registro dos dados cinemáticos foi utilizada uma câmera digital marca Sony. A câmera ficou suspensa a uma al tura de 1,51 m do tampo da mesa, sobre a qual foi realizada a tarefa solicitada, de forma a permitir a visual ização do participante e da tarefa por el e executada a partir de uma vista superior.

O registro dos dados cinemáticos foi realizado por meio defilmageme a análise foi real izada com o auxílio de um programa para análise de movimento, Kavideo. A análise computadorizada do movimento pelo programa Kavideo foi realizada em duas dimensões (2D).

Marcadores reflexivos auto-adesivos foram col ocados sobre os pontos anatômicos do membro superior dominante de cada participante, necessários para posterior digitalização e análise do movimento: 1) na proeminência óssea do acrômio; 2) no epicôndilo lateral da ulna; 3) no processo estilóide da ulna e do rádio; 4) nas articulações metacarpofalangeanas do pol egar, dedo indicador ededo médio.

Para posterior reconstrução dos pontos no espaço, foram determinadas as medidas de alcance máximo de cada participante para o cálculo da escala de pólos, solicitada pelo programa como referência. De acordo com Audi (2006), a escala de pólos é a distância linear entre dois pontos previamente mensurados, cujo valor ésolicitado pelo programa deanálise demovimento Kavideo, queserve como fator deconversão das coordenadas ortogonais XY. As medidas da escala de pólos compreendem os limites extremos entre o al cance máximo do participantee a raiz da articulação do ombro do membro superior dominante, utilizado para a execução do movimento. de pólos (Tabela 1).

Assim, o al cance máximo de cada participantefoi usado para sua escala

Tabela 1 - Medida da escala de pólos calculada para cada participante

\begin{tabular}{cc} 
Participantes & Escala de pólos \\
\hline P1 & $69 \mathrm{~cm}$ \\
P2 & $79 \mathrm{~cm}$ \\
P3 & $78 \mathrm{~cm}$ \\
P4 & $48 \mathrm{~cm}$ \\
P5 & $49 \mathrm{~cm}$ \\
P6 & $74 \mathrm{~cm}$ \\
P7 & $68 \mathrm{~cm}$ \\
P8 & $69,50 \mathrm{~cm}$ \\
P9 & $52 \mathrm{~cm}$ \\
P11 & $51 \mathrm{~cm}$ \\
\hline
\end{tabular}


A análise cinemática envolveu a mensuração do desempenho dos movimentos realizados durante a execução da tarefa solicitada ao participante. Assim, as medidas fornecidas pelo programa Kavídeo usadas nesseestudo foram: 1) frames ou quadros por segundo, utilizados durante a execução da tarefa; 2) velocidade escal ar total.

\subsection{Análise cinemática}

Uma placa de vídeo do computador permitiu a captura das imagens por meio de uma entrada compatível com a filmadora utilizada. Desta forma, após a filmadora ter sido conectada ao computador, foi possível capturar as imagens por meio do programa para análise do movimento Kavídeo (AUDI, 2006).

A pós a captura das imagens foi realizada a formatação dos frames e conversão gráfica. Por intermédio do programa Kavídeo, foi possível reconstruir o movimento a partir dos marcadores em cada ponto anatômico selecionado para o estudo e pela medida da escala de pólos. A digitalização foi realizada por meio do cursor do mouse sobre o centro de cada marcador eas coordenadas de cada ponto foram armazenadas em um arquivo de dados do computador para posterior exportação para o programa Microsoft Excel.

Por meio da utilização do programa Kavídeo, foi possível obter dados para esse estudo que compreenderam as quantidades de frames obtidos durantea execução da tarefa e a velocidade escalar. Para chegar ao cálculo do tempo despendido para a execução da tarefa, dividiu-se a quantidade de frames por 60. Utilizou-se a seguinte equação:

$$
t=\frac{n^{\circ} \text { totaldeframes }}{60}
$$

Para o cálculo da velocidade escalar média, foi feita a divisão da velocidade escal ar total pela quantidade de frames.

\subsection{Estatística}

Devido à natureza dos dados foi realizada a análiseestatística descritiva por meio de média, desvio-padrão, valor máximo e mínimo.

A análise da influência da flexibilidade do assento foi realizada por meio de teste estatístico não-paramétrico, Teste de Wilcoxon. $O$ valor adotado foi $p=0,05$.

\section{RESULTADOS}

Os resultados foram apresentados na seguinte seqüência: 1) velocidade média durantea execução da tarefa; 2) tempo despendido para a execução da tarefa. 


\subsection{Velocidade média dURANTE A EXECUÇão da tAREFA}

$\mathrm{N}$ a Tabela 2 são apresentados os dados referentes à velocidade média do membro superior dominante durante a execução da tarefa de ida até o ponto central. No assento de lona foram encontrados os seguintes valores: média 0,174 \pm 0,051 metros por segundos (m/ s); mínimo 0,093 m/ s e máximo 0,249 m/ s. No assento de madeira os valores obtidos foram: média $0,187 \pm 0,043 \mathrm{~m} / \mathrm{s}$; mínimo $0,106 \mathrm{~m} / \mathrm{s}$ e máximo $0,240 \mathrm{~m} / \mathrm{s}$.

Tabela 2 - Resultado da análise da velocidade média $(\mathrm{m} / \mathrm{s})$ do membro superior durante a execução da tarefa de ida até o ponto central.

\begin{tabular}{lcc}
\hline & lona & madeira \\
\hline Média & 0,174 & 0,187 \\
Desvio padrão & 0,051 & 0,043 \\
Mínimo & 0,093 & 0,106 \\
Máximo & 0,249 & 0,240 \\
Coeficiente de variação & 0,30 & 0,230 \\
\hline
\end{tabular}

A análise da comparação dos resultados obtidos nos diferentes assentos, por meio do teste deWilcoxon, constatou que não existia significância na diferença estatística $(p=0,45)$. Desta forma, a velocidade média do membro superior dominanteduranteexecução da tarefa deida atéo ponto central não foi influenciada pel o tipo de assento utilizado, Iona ou madeira.

Os dados referentes à velocidademédia do membro superior dominante durante o movimento de retorno mostraram que quando foi utilizado o assento de Iona a média foi $0,201 \pm 0,054 \mathrm{~m} / \mathrm{s}$, enquanto no assento de madeira a média foi de $0,212 \pm 0,076 \mathrm{~m} / \mathrm{s}$ (Tabela 3).

Tabela 3 - Resultado da análise da velocidade média $(\mathrm{m} / \mathrm{s})$ do membro superior durantea execução da tarefa de retorno ao ponto inicial para cada tipo deassento.

\begin{tabular}{lcc}
\hline & lona & madeira \\
\hline Média & 0,201 & 0,212 \\
Desvio padrão & 0,054 & 0,076 \\
Mínimo & 0,134 & 0,118 \\
Máximo & 0,302 & 0,333 \\
Coeficiente de variação & 0,270 & 0,360 \\
\hline
\end{tabular}

A análise da comparação dos resultados obtidos nos diferentes assentos, por meio do teste de Wilcoxon, constatou que não existia significância na diferença estatística $(p=0,59)$. Desta forma, a velocidade média do membro superior dominante durante execução da tarefa de retorno ao ponto inicial não foi influenciada pelo tipo de assento utilizado, lona ou madeira. 


\subsection{TEMPO DESPENDIDO PARA A EXECUÇÃo dA TAREFA}

$\mathrm{Na}$ Tabela 4 encontram-se os dados referentes ao tempo despendido em segundos (s) para a execução da tarefa de ida atéo ponto central nos diferentes tipos deassento. N o assento de lona os valores obtidos foram: média 3,366 $\pm 1,766 s$; mínimo 1,430 s e máximo 7,000s. No assento de madeira; média 2,457 $\pm 1,096 \mathrm{~s}$; mínimo 1,096s e máximo 5,300s. Observa-se que os participantes realizaram a atividade em um tempo menor quando foi utilizado o assento de madeira.

Tabela 4 - Resultado da análise do tempo (s) despendido durante a execução da tarefa de ida até o ponto central.

\begin{tabular}{lcc}
\hline & lona & madeira \\
\hline Média & 3,366 & 2,457 \\
Desvio padrão & 1,766 & 1,096 \\
Mínimo & 1,430 & 1,300 \\
Máximo & 7,000 & 5,300 \\
Coeficiente de variação & 0,530 & 0,450 \\
\hline
\end{tabular}

A análise da comparação dos resultados obtidos nos diferentes assentos, por meio do teste de Wilcoxon, constatou que existia significância na diferença estatística $(p=0,03)$. Desta forma, o tempo despendido durante execução da tarefa de ida ao ponto central foi influenciado pelo tipo de assento utilizado.

Os dados referentes ao tempo despendido em segundos durante a execução da tarefa deretorno ao ponto inicial encontram-sena Tabela 5. No assento de lona os valores obtidos foram: média 2,363 $\pm 0,235$ s; mínimo 1,430s; máximo 4,000 s. No assento de madeira: média 1,969 $\pm 0,786 s ;$ mínimo 1,270 s e máximo 3,930s. Durante a realização desta tarefa o tempo despendido, também, foi menor quando se utilizou o assento de madeira.

Tabela 5 - Resultado da análise do tempo (s) despendido durante a execução da tarefa de retorno ao ponto inicial para cada tipo de assento.

\begin{tabular}{lcc}
\hline & Lona & madeira \\
\hline Média & 2,363 & 1,969 \\
Desvio padrão & 0,235 & 0,786 \\
Mínimo & 1,430 & 1,270 \\
Máximo & 4,000 & 3,930 \\
Coeficiente de variação & 0,330 & 0,400 \\
\hline
\end{tabular}

A análise da comparação dos resultados obtidos nos diferentes assentos, por meio do teste de Wilcoxon, constatou que existia significância na diferença estatística $(p=0,05)$. Desta forma, o tempo despendido durante execução da tarefa de retorno foi influenciado pelo tipo de assento utilizado. 


\section{Discussão}

A aquisição de um equilíbrio postural eficiente, na posição sentada ou em pé, é uma das maiores dificuldades encontradas pela criança com paralisia cerebral.

Equilíbrio postural sentado pode ser definido como a habilidade de um indivíduo na posição sentada em manter o deslocamento do centro de massa sobre uma base desustentação durantea execução de diferentes atividades iniciadas pelo próprio individuo, esem cair excessivamente(DEAN ; SHEPHERD; ADAMS, 1999). Para estes autores, al cançar alvos em várias distâncias do corpo éuma ação comum que perturba o equilíbrio, uma vez que envolve interações complexas entre o braço, o tronco superior e a base da sustentação que é fornecida por meio da pelve e pernas no assento e pel os pés no assoalho.

O déficit no equilíbrio postural interfere na aquisição de habilidades motoras, na interação social e na comunicação, pois dificulta o desempenho funcional da criança com paralisia cerebral nas atividades cotidianas, tais como: alcançar um objeto, segurar um lápis, escrever, entre outras.

Vários estudos têm mostrado que um posicionamento sentado adequado melhora a estabilidade e o alinhamento postural, além de favorecer o desempenho com os membros superiores (NWAOBI, 1987; GREEN; NELHAM, 1991; MYHR; VON WENDT, 1991; MCCLEN A GHAN; THOMBS; MILNER, 1992; REID 1996; BRACCIALLI, 2000; SHEN; KANG; WU, 2003).

Para a criança com paralisia cerebral manter uma postura sentada estável eal inhada, geral mente, ela necessita de um recurso de tecnologia assistiva adequado. Comumente, profissionais que atendem estas crianças em clínicas ou na escola indicam aos familiares ou cuidadores a aquisição de um mobiliário adaptado. No entanto, ainda existem controvérsias sobre qual a melhor postura da criança para favorecer o desempenho funcional e sobre qual o mobiliário mais adequado em cada situação.

A pesar de ser incontestável a importância da aquisição e do uso de dispositivos para auxiliar no equilíbrio postural de crianças com paralisia cerebral, vários estudos têm mostrado que grande parte dos dispositivos adquiridos é abandonada pelo usuário entreo primeiro ano eo quinto ano de uso, eal guns não chegam nem mesmo a ser utilizado (PHILLIPS; ZHAO, 1993; GOODM AN ; TIENE; LUFT, 2002; SCHERER, 2002; VERZA et al., 2006).

Desta forma, um profissional ao prescrever um mobiliário deve ter clareza sobre os objetivos a serem alcançados com aquele dispositivo, seus benefícios e suas limitações.

Os dados desteestudo indicaram que, apesar denão haver significância estatística, a velocidade registrada durante a execução da atividade no assento de madeira foi ligeiramente maior que a registrada no assento de lona. 
Os participantes do estudo gastaram em média um tempo maior durante a execução do movimento de ida até o ponto central do que na realização do movimento de retorno ao ponto inicial. Chang et al. (2005) relataram que as crianças com paralisia cerebral espástica, quando realizaram um movimento de al cance de precisão tinham um tempo de movimento prolongado.

Os resultados desse estudo em relação ao tempo despendido para a execução da atividade corroboraram com os achados de Dean, Shepherd e Adams (1999) que descreveram que a distância a ser percorrida e a tarefa executada tem influência no tempo despendido no movimento da mão. Para Shumway-Cook e Woollacott (2003) o tempo e as estratégias para a programação dos movimentos variam com o objetivo da tarefa. Quando o movimento exige uma parada exata que requer do indivíduo uma ação defrenagem, como no movimento deencaixerealizado neste estudo, o tempo despendido para a realização do movimento é maior.

Os dados do estudo indicaram que quando os participantes utilizaram um assento demadeira às atividades eram realizadas em um período detempo menor.

Estudo realizado com indivíduos saudáveis Gaudez, Richardson e Lê Bosec (2004) concluíram que os ajustes e o desempenho dinâmico postural de uma tarefa dependem da densidade da superfície de apoio. Os resultados apresentados pelos autores sugerem queo ambientefísico influencia a organização posturo-cinética.

Durante a execução de uma tarefa voluntária os diferentes segmentos do corpo devem ser deslocados de uma maneira coordenada para assegurar a estabilização do corpo e a manutenção do equilíbrio. Deve haver uma ação coordenada de cabeça, pélvis, tronco, membros superiores e inferiores durante o movimento de alcance.

O assento delona, por ser uma superfíciemais instável queo assento de madeira, parece favorecer uma maior instabilidade postural e déficit de equilíbrio. A estabilidadedo tronco em uma superfícieinstável depende da habilidadedealinhar a projeção de centro de massa com o centro da rotação da superfície de sustentação, adicionado a isto, o sistema nervoso central, também tem quecontrolar forças inerciais geradas pelos movimentos do tronco (LANZETTA et al., 2004)

Desta forma, a instabilidade pélvica provocada pelo uso de um assento de lona pode exigir um maior número de deslocamento do centro de gravidade o que gera um déficit de equilíbrio e conseqüentemente resultará em execução de tarefas em um período de tempo maior. Para Aissaoui et al. (2001) o déficit do controle do equilíbrio sentado pode limitar o desempenho da tarefa.

\section{CONCLUSÃO}

Os resultados encontrados nesteestudo mostraram quea flexibilidade do assento é uma variável importante a ser considerada na prescrição e indicação de mobiliários adaptados para al unos com paralisia cerebral espástica. 
A análise cinemática indicou que o tempo despendido para executar as atividades era maior quando foi utilizado o assento de lona.

Conclui-se que cadeiras de rodas ou adaptadas com assento de lonas não são os mobiliários mais adequados para serem utilizados por alunos com paral isia cerebral durante suas atividades escolares e ou atividades de vida diária.

A pesar deste tipo de mobiliário ser comumente encontrado em salas especiais ou salas regulares para posicionamento de alunos com deficiência física, elas são inadequadas para este tipo de atividade, pois parece aumentar a instabilidade postural e piorar o desempenho durante as atividades.

\section{Referências}

AISSA OUI, R. et al. Effect of seat cushion on dynamic stability in sitting during a reaching task in wheel chair users with paraplegia. A rch Phys M ed Rehabil, v.82, p.274-81, 2001.

AUDI, M. Estudo comparativo do comportamento motor de membro superior em encefal opatas que fazem uso de pulseira estabilizadora. 2006. 112 f. Dissertação (Mestrado em Educação) Faculdade de Filosofia e Ciências, Universidade Estadual Paulista, 2006.

BRACCIALLI, L. M. P. Influência da utilização do mobiliário adaptado na postura sentada de indivídu os com paralisia cer ebral espástica. 2000. 100f. Tese (Doutorado em Educação Física). Faculdade de Educação Física - Universidade Estadual de Campinas, Campinas, 2000.

CHANG, J. J. et al. Kinematical measure for spastic reaching in children with cerebral palsy. Clinical Biomechanics, v.20, p.381-388, 2005.

DEAN, A.; SHEPHERD, R.; ADAMS, R. Sitting bal ancel: trunk-arm coordination and the contribution of the lower limbs during self-paced reaching in sitting. $G$ ait and Posture, v.10, p.135-146, 1999.

EITZEN , I. Pressuremapping in seating: a frequency analysis approach. A rchives of P hysical M edicine and Rehabilitation, v.85, p.1136-1140, 2004.

GAUDEZ, C.; RICHARDSON, J.; LEE BOSEC, S. Influence of characteristics of support surface on postural dynamics in isometric force production. Premus, v.II, p.385-386, 2004.

GOODMAN, G.; TIENE, D; LUFT, P. A doption of A ssistiveTechnology for computer access among college students with disabilities. D isability and R ehabilitation, v.24, n.1/2/3, p. 8092, 2002.

GREEN , E. M.; NELHAM, R. L. Development of sitting ability, assessment of children with a motor handicap and prescription of appropriate seating systems. P rosthetics and 0 rthotics International, v.15, p.203-216, 1991.

JAROSZ, E. Determination of the workspace of wheelchair users. International Journal of Industrial Ergonomics, v.17, p.123-133, 1996.

KOCHHANN , A. R. S.; CA NALI, N.; SERA FIM , M. A. P. Comparação de picos de pressão em assento flexível em portadores de lesão medular eindivíduos normais: uma avaliação por interface de pressão. A cta Fisiátrica, v.11, n.3, p.95-100, 2004.

LANZETTA, D. et al. Trunk control in unstablesitting posture during functional activities in healthy subjects and patients with multiplesclerosis. A rch P hys M ed Rehabil, v.85, p.27983, 2004. 
MCCLENAGHAN, B.A., THOMBS, L., MILNER, M. Effects of seat-surface inclination on postural stability and function of the upper extremities of children with cerebral palsy. D evelopmental M edicineand Child N eurology, n. 34, p.40-48, 1992.

MYHR, U., VON WENDT, L. Reducing spasticity and enhancing postural control for the creation of a function sitting position in children with cerebral palsy: a pilot study. Phys Theor and Pract, vol.6, p.65-76, 1990.

MYHR, U. et al. Five-year follow-up of function sitting position in children with cerebral palsy. D ev. M ed. Child N eurol., v.37, p.587-596, 1995.

MYHR, U.; WENDT, L.V. Improvement of fuctional sitting position for children with cerebral palsy. D evelopmental M edicine and Child N eurology, v.33, p.246-256, 1991.

MYHR, U.; WENDT, L. V. Influence of different sitting positions and abduction orthoses on leg muscle activity in children with cerebral palsy. Developmental M edicine and Child N eurology, v.35, n.10, p.870-880, 1993.

NOWAK, E. The role of antropometry in design of work and life environments of the disabled population. International Journal of Industrial Ergonomics, v.17, p.113-121, 1996.

NWAOBI, O. M. Seating orientations and upper function in children with cerebral palsy. Physical Therapy, v.67, n.8, p.1209-1212, 1987.

NWA OBI, O. M. et al. Electromyographic investigation of extensor activity in cerebralpalsied children in different seating positions. D evel opmental $M$ edicineand $C$ hild $\mathrm{N}$ eurology, v. 25, p.175-183, 1983.

PHILLIPS, B.; ZHAO, H. Predictors of assistive technology abandonment. A ssistive Technology, v. 5, p. 36-45, 1993.

REID, D. T. The effects of the saddle seat on seated postural control and upper-extremity movement in children with cerebral palsy. D evel opmental M edicine and C hild N eurology, v.38, p.805-815, 1996.

REID, D. T.; SOCHANIWSKYJ, A.; MILNER, M. Instrumentation and a Protocol for Quantification of Upper-Limb Movement of Children with and without cerebral palsy in two sitting positions. Journal of N eurology Rehabilitation, v.6, p.25-34, 1992.

SCHERER, $M$ The change in emphasis from people to person: introduction to the special issue on Assistive Technology. D isability and R ehabilitation, v 24, n. 1/ 2/ 3, p.1-4, 2002.

SEEGER, B.R.; CAUDREY, D.J.; O'MARA, N.A. Hand function in cerebral pal sy: the effect of hip-flexion angle. D evelopmental M edicine and Child N eurology, v.26, p.601-606, 1984.

SHEN, I., KANG, S. M, WU, C. Comparing the effect of different design of desks with regard to motor accuracy in writing performance of students with cerebral palsy. A pplied ergonomics, n.34. p.141-147, 2003.

SHUMWAY-COOK, A; WOOLLA COTT, M. H. Controlemotor. São Paulo: Manole, 2003.

VERZA, R. et al. An interdisciplinary approach to evaluating the need for assistive technol ogy reduces equipment abandonment. M ultipleSclerosis, v.12, p.88-93, 2006.

WASHINGTON, $K$ et al. The effects of a contoured foam seat on postural alignment and upper-extremity function in infants with neuromotor impairments. Physical Therapy, v.82, n.11, p.1064-1076, 2002.

Recebido em 17/ 09/2007

Reformulado em 28/ 02/ 2008

A provado em 30/ 03/ 2008 